\title{
Haptic parallelity perception on the frontoparallel plane: The involvement of reference frames
}

\author{
Robert Volcic, ASTRID M. L. KAPPERS, AND JAN J. KOENDERINK \\ Utrecht University, Utrecht, The Netherlands
}

\begin{abstract}
It has been established that spatial representation in the haptic modality is subject to systematic distortions. In this study, the haptic perception of parallelity on the frontoparallel plane was investigated in a bimanual matching paradigm. Eight reference orientations and 23 combinations of stimulus locations were used. The current hypothesis from studies conducted on the horizontal and midsagittal planes presupposes that what is haptically perceived as parallel is a product of weighted contributions from both egocentric and allocentric reference frames. In our study, we assessed a correlation between deviations from the veridical and hand/arm postures and found support for the role of an intermediate frame of reference in modulating haptic parallelity on the frontoparallel plane as well. Moreover, a subject-dependent biasing influence of the egocentric reference frame determines both the reversal of the oblique effect and a scaling effect in deviations as a function of bar position.
\end{abstract}

In order to interact effectively with the world, people must be able to reach for and manipulate objects in their immediate environment. These acts require that an agent deal with the spatial relations of various objects with respect to the body. The space in which these relations are coded and in which the agent acts is a product of multiple perceptual modalities. When sensory input is limited to the single modality of touch, the perceptual space can be referred to as haptically perceived space or haptic space. The term haptic perception refers to tactual perception in which both the cutaneous sense and kinesthesis convey information about distant objects and events (Loomis \& Lederman, 1986).

Although the idea appears counterintuitive, perceptual spaces (visual, auditory, or somatosensory) are typically structured differently from the corresponding physical spaces, and thus perceptual spatial judgments are generally nonveridical. Similarly, several earlier studies have established that the perception of haptic space, and consequently the perception of spatial relations, is far from veridical (Blumenfeld, 1937; Hermens, Kappers, \& Gielen, 2006; Kappers, 1999, 2002, 2003, 2004, 2005; Kappers \& Koenderink, 1999; Newport, Rabb, \& Jackson, 2002; Zuidhoek, Kappers, van der Lubbe, \& Postma, 2003). These studies were performed on the horizontal, midsagittal, and frontoparallel planes and involved numerous stimulus locations in unimanual or bimanual conditions. In the various conditions, several tasks were undertaken, such as the parallelity, collinearity, mirroring, and pointing tasks. All of these tasks were performed on the horizontal plane, whereas only the parallelity task was performed on other planes. In the parallelity task, in which participants match the orientations of two bars, a common outcome has been repeatedly confirmed, that participants produce large systematic deviations from physical parallelity. In the parallelity task, systematic directional errors of more than $90^{\circ}$ can occur. Moreover, the magnitude of these deviations is clearly subject dependent.

Encoding the location and orientation of an object implies the existence of a frame of reference in which space coordinates can be defined. Classically, a distinction is made between allocentric and egocentric frames of reference (Berthoz, 1991; Klatzky, 1998); the latter can be fixed to the hand (Carrozzo \& Lacquaniti, 1994; Paillard, 1991), the arm (Flanders \& Soechting, 1995; Soechting \& Flanders, 1992, 1993), or the body (Luyat, Gentaz, Corte, \& Guerraz, 2001; Millar \& Al-Attar, 2004). Moreover, ample evidence supports the idea that specific attributes are defined in neither an allocentric nor an egocentric frame of reference, but in a frame that is intermediate to those two (Carrozzo \& Lacquaniti, 1994; Flanders \& Soechting, 1995; Luyat et al., 2001; Paillard, 1991; Soechting \& Flanders, 1992, 1993). Accordingly, the experimental results obtained in the parallelity task can be described by applying the reference-frame-based model devised by Kappers (2002, 2003, 2004, 2005). Specifically, an interaction between two reference frames, allocentric and egocentric, has been hypothesized, with the coordinate systems of the two frames having different origins. The origin of the allocentric reference frame can be considered to be independent of the actual position of the perceiver, since it is linked to the external space, is aligned with gravity, and defines spatial relations with respect to elements of the environment. It should be noted that the allocentric reference frame is defined as being anchored in the external space, even though initially it inevitably has to

R.Volcic, r.volcic@phys.uu.nl 
be defined via egocentric experiences. On the other hand, the origin of the egocentric reference frame is assumed to be coupled to the body or to a specific body part of the perceiver, like the arm or the hand. Therefore, changes in the perceiver's position relative to the allocentric reference frame lead to modifications of the egocentric reference frame. Accurate performance in the parallelity task could only be achieved if the haptic perception of parallelity relied on the allocentric reference frame, because the task itself is defined in allocentric terms. On the other hand, if haptic parallelity were based on the egocentric frame only, factors such as the rotation of the hand involved in a specific task, for example, would determine the amount of deviation from what is physically parallel. In practice, what feels haptically parallel is always intermediate between parallelity as defined by the allocentric frame and by the egocentric frame. In other words, haptic parallelity is determined by a biasing influence of the egocentric frame of reference. Hence, the experimental results can be interpreted as being weighted averages of the contributions of the two reference frames, and the magnitude of deviations is determined by the degree to which the allocentric and egocentric reference frames combine with each other. Hermens et al. (2006) have proposed an alternative hypothesis suggesting that the deviations could be explained by errors in transferring the reference orientation from one hand to the test bar position on the other hand. In contrast, several results, among them the correlations between deviations and hand orientations found by Kappers (2005), are not explainable by the transfer-ofinformation hypothesis and seem to disprove it.

In addition, a series of studies (Hermens et al., 2006; Kappers, 1999, 2002, 2003, 2004; Kappers \& Koenderink, 1999) have explored the effect of the reference bar orientation on systematic deviations - that is, on errors in terms of the constant directional error (accuracy). The major observation has been that the biasing influence of the egocentric reference frame again plays a crucial role. Participants with smaller average deviations, who therefore relied more upon the allocentric representation, performed more poorly (i.e., had larger deviations) at oblique than at vertical and horizontal orientations, a phenomenon known as an oblique effect. Conversely, participants with larger average deviations showed a reverse oblique effect, performing better (i.e., having smaller deviations) at orientations that were oblique in an allocentric reference frame but were vertical or horizontal accordingly to their egocentric reference frame. Newport et al. (2002) reported another observation concerning the dependence on reference orientation in a slightly different experimental design. By studying only the effect of diverse oblique orientations, they found an almost linear increase in deviations as the sensing arm assumed more extreme postures.

Another branch of haptic perception studies (see below) has focused on the oblique effect by looking at the variability of settings instead of at accuracy. Variability and accuracy are independent measures, and variability has been observed to be greater at oblique orientations than at vertical and horizontal orientations. Many factors that could have some bearing on the oblique effect have been considered. For instance, some have suggested that the effect arises as a result of visual experience and imagery (Appelle \& Countryman, 1986; Appelle \& Gravetter, 1985). On the other hand, studies involving the use of different planes and different body and head tilts have suggested that gravitational cues play a certain role (Gentaz \& Hatwell, 1996; Luyat, et al., 2001). Furthermore, the existence of the oblique effect has been ascertained both in children and blind adults (Gentaz \& Hatwell, 1995, 1998; Gentaz \& Streri, 2004). In particular, Gentaz and Hatwell (1998) showed the presence of the effect in both early- and late-blind people, refuting the hypothesis that visual experience plays a major role. In addition, the persistence of the effect has been observed in conditions that involved delayed reproductions and memory constraints (Gentaz \& Hatwell, 1999; Lechelt \& Verenka, 1980). Finally, the oblique effect has also been examined in intramodal and crossmodal conditions involving the haptic, visual, and somatovestibular systems (Gentaz et al., 2001; Lechelt, Eliuk, \& Tanne, 1976; Lechelt \& Verenka, 1980). Whereas the latter studies have always examined only a few bar locations on a specific plane, the haptic space studies have concentrated on the systematic deviations that occur over the whole region of space within the reach of the hands, defined as peripersonal or manipulatory space (Lederman, Klatzky, Collins, \& Wardell, 1987).

The purpose of our study was to focus on the perception of haptic space on the frontoparallel plane. Blindfolded participants had to rotate a test bar in such a way that it felt parallel to a reference bar. Bars were displaced laterally with respect to the body midline and were easily reachable with the extended arms. The amount and direction away from physical parallelity and the orientation of the hand at different locations on the plane were measured. Since we hypothesized that hand/arm orientation is probably interconnected with the orientation of the egocentric reference frame, we expected the amount of deviation at a specific location to be at least partially correlated with the change in hand orientation. Recently, Hermens et al. (2006) conducted a study on the frontoparallel plane in which only a few bar locations were used, and consequently in which only a few hand/arm orientations were involved. This experimental limitation has probably constrained the reference-frame-based interpretation of the results of the parallelity task, because no correlation was found between hand orientation and deviation.

The primary aim of the present article is to establish whether systematic deviations from what is physically parallel can be detected on the frontoparallel plane, and whether these deviations are comparable with those found in studies performed on the horizontal and midsagittal planes. Exploratory movements could be influenced by the plane in which a task is executed; therefore, it is of fundamental importance to compare performance on the three primary orthogonal planes. Only a thorough exploration of the patterns of deviations on different planes can lay the foundation for a comprehensive explanation of the distortions that occur in the haptic perception of space. Second, by monitoring numerous bar locations, we wanted to study the role that allocentric and egocentric 
reference frames play in determining the pattern of errors. Specifically, we wanted to find out whether it would be possible to describe our results by applying the referenceframe-based model derived from earlier studies (Kappers, 2002, 2003, 2004, 2005). Our third aim, which was no less important, was to find out more about orientation dependency by taking into account a finely distributed set of possible matching orientations. The effect can be investigated over a considerable number of stimulus locations in our paradigm, making it possible to observe eventual modifications in the perception of orientation on the frontoparallel plane. Finally, since previous studies have shown that more egocentrically characterized participants display a reverse oblique effect, it will be significant to see whether the same kind of reversal occurs in the present research.

\section{METHOD}

\section{Participants}

The 8 undergraduates ( 7 male, 1 female) who took part in this experiment were remunerated for their efforts. None of them had any prior knowledge of the experimental design and the task. The handedness of the participants was assessed by means of a standard questionnaire (Coren, 1993), and all were right handed, except for 1 (E.W.) who was ambidextrous and 1 (L.W.) who was left handed.

\section{Apparatus}

The setup consisted of a large, vertically positioned whiteboard that could be adjusted in height. Protractors with a radius of $12 \mathrm{~cm}$ were printed on the whiteboard with their centers $30 \mathrm{~cm}$ apart, both horizontally and vertically. A subset of 10 protractors (indicated by the filled circles in Figure 1) were used in this experiment. An aluminum bar, with an axle in the middle, could be inserted in the center of each protractor and rotated freely. Small magnets were attached under the bar to prevent accidental rotations. Two bars with a length of $20 \mathrm{~cm}$ and a diameter of $1 \mathrm{~cm}$ were used as the test and reference bars. The bars had an arrow-shaped end on one side that allowed the reference bar orientation and the test bar orientation to be read off with an accuracy of $0.5^{\circ}$.

Pictures of hand orientations were taken with a digital camera (Canon Digital Ixus 400). This camera produced jpeg files with a resolution of $2,272 \times 1,704$ pixels.

\section{Design}

The reference bar was placed in a total of 10 different locations. Five of the locations were distributed on the left side, and 5 in symmetrically located positions on the right side of the board (filled circles in Figure 1). The test bar was always located on the contralateral side of the participant from the reference bar (i.e., on the right side when the reference bar was on the left, and vice versa). In total, there were 23 combinations of locations of the two bars. ${ }^{1}$ This specific set of stimuli was adopted in order to allow a sufficiently large number of location combinations in which participants could assume different hand/arm orientations without changing the posture of their elbow joints. As a comparison, in Hermens et al.'s (2006) study on the frontoparallel plane, bars were positioned at only four locations: $(-45,30),(-45,-60),(45,30)$, and $(45,-60)$. For each combination, the reference bar in our experiment was set at one of eight orientations, from $0^{\circ}$ to $157.5^{\circ}$ in steps of $22.5^{\circ}\left(90^{\circ}\right.$ being the vertical orientation and $0^{\circ}$ pointing horizontally to the body midline); the test bar was oriented randomly. The reference bar was located either on the left or on the right side of the body midline. The order of the 368 trials in a block ( 23 combinations of bar locations $\times 8$ orientations $\times 2$ reference bar locations) was randomized for each participant. The block of 368 trials was repeated three times with different randomizations, for a total of 1,104 trials per participant.

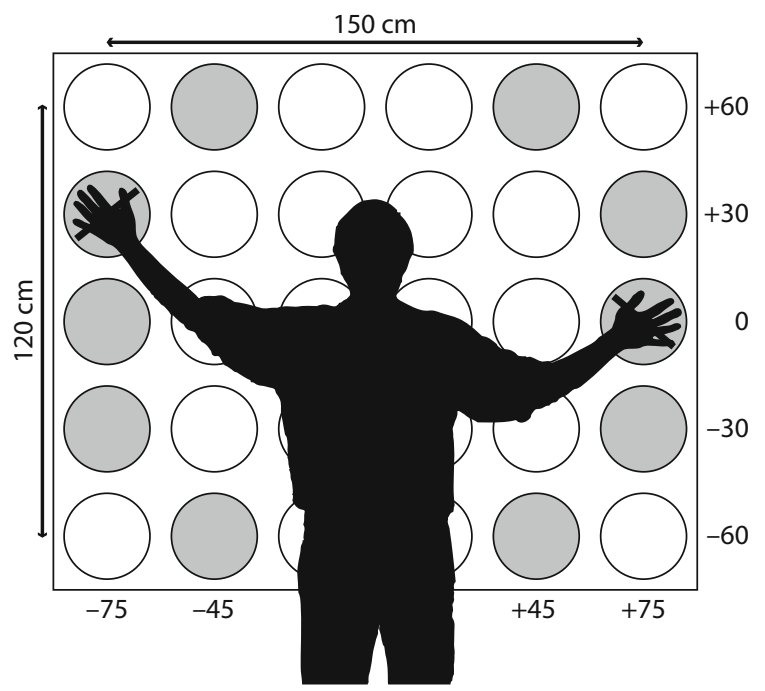

Figure 1. Schematic representation of a blindfolded participant performing the parallelity task on the frontoparallel setup. The orientation of the bars represents a realistic final setting. The filled circles represent the locations at which the reference and test bars could be fixed. The two bars were always positioned on opposite sides relative to the body midline.

\section{Procedure}

Blindfolded participants had to perform a bimanual parallelity task. The participants were placed in front of the whiteboard at a distance of about $30 \mathrm{~cm}$ from the board, with the body midline aligned with respect to the midpoint of the setup. The standing position was specified by a $30 \times 30 \times 2 \mathrm{~cm}$ platform attached to the floor. From this position, all locations on the whiteboard were within easy reach; therefore, no displacement of the body was either necessary or allowed. The height of the whiteboard was adjusted for each participant in such a way that the shoulders were at the same distance from the upper and lower bar locations (i.e., at height 0 in Figure 1).

The experimenter fixed the positions and orientations of the bars. Subsequently, the hands of a participant were placed on the bars, first on the reference bar and then on the test bar. Both bars were touched simultaneously for the whole duration of each trial; the left hand always touched the left bar, the right hand the right bar. The participants were instructed to rotate the test bar in such a way that they felt it to be parallel to the reference bar. No specific instruction was given about how to explore the two bars, and participants were allowed to use their fingers, palms, and hands to touch the bars either statically or dynamically. They had $10 \mathrm{sec}$ to explore the bars and orient the test bar, which appeared to be a more than adequate amount of time. An electronic digital timer measured the time, with a beep signaling when it had run out. Participants then removed their hands from the setup and the experimenter wrote down the measurement before starting with the next trial. No feedback was given on their performance. The experimental sessions ended after $1 \mathrm{~h}$, in order to prevent fatigue for the participants, and were performed on separate days. Participants took on average $8-9 \mathrm{~h}$ to complete all of the sessions. They did not have the chance to see the setup until all sessions were over, because it was covered both before and after each session.

After completion of the parallelity task, one more experimental session took place. In order to monitor the influence of hand orientation, the experimenter measured the orientations of both the left and right hands of each participant for each position employed in the parallelity task. We did this because the orientation of the hand indicates the orientation of the egocentric frame of reference fixed to the hand. In addition, the forearm and the hand were kept aligned throughout this experimental session. This method was previously 

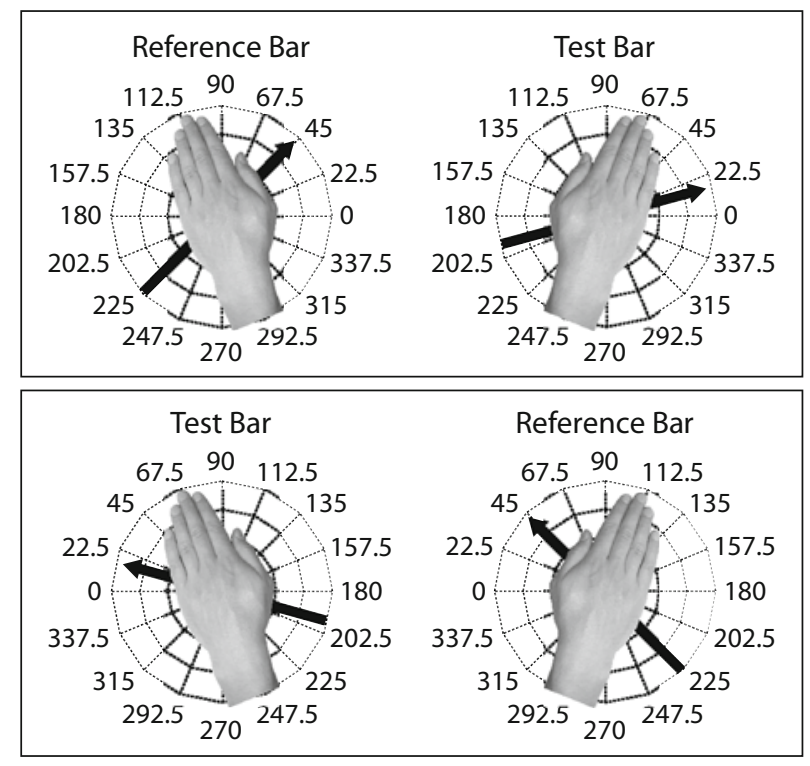

Figure 2. The orientation of the bar is defined by its location relative to the body midline. The $0^{\circ}$ orientation of the reference bar always points horizontally to the projection on the board of the body midline. For the left-located reference bar, the degree of orientation increases in a counterclockwise direction, whereas for the right-located reference bar, it increases in a clockwise direction.

utilized by Kappers (2005), who demonstrated a correlation between deviations and hand orientations. For this final session, participants resumed the standing position in front of the board, but with no bars attached to it. They were asked to place one hand on the board in a natural way (with no radial or ulnar deviation) at a position indicated by the experimenter. During this session, they were allowed to see the setup in order to place their hand at the center of the protractor in the requested position. They were also asked to hold their extended fingers close to each other (finger adduction), as can be seen in Figure 2. The requested positions corresponded to the locations at which the bars had been situated during the parallelity task, and their order was randomized. When each participant's hand touched the board, a picture was taken with a digital camera. In total, three pictures were taken for each predetermined position. Hand orientation was defined as the pointing direction of the middle finger when the hand was lying on the surface of the board. Measurements of hand orientation were then extracted from all the pictures and averaged over the three repetitions. Participants took on average a half hour to complete this session.

\section{Data Analysis}

The studies concerning haptic space perception have established that deviations vary in a systematic way. Deviations occur in a counterclockwise direction when the reference bar is on the right of the test bar, whereas they occur in a clockwise direction when the reference bar is on the left of the test bar. Such deviations are defined as the orientation of the left bar minus the orientation of the right bar; thus, the deviation specifies both the direction and the size of the error. It follows that positive values are assigned to deviations in the expected direction, and negative values to deviations in the opposite direction.

In the present study, the reference bar was located either on the left or the right side of the body midline. In order to analyze the influence of the reference bar orientation, it was advantageous to combine data from the left and right reference bar conditions. To allow this data aggregation, reference orientations needed to be defined in such a way that the relation between the sensing hand and a specific orientation of the reference bar would be identical for both the left and right reference bar conditions. Figure 2 represents the method we used for codifying the data. The orientation of the reference bar was defined by its location relative to the body midline. When the reference bar was located on the left side of the body midline, the $0^{\circ}$ reference orientation was set on the positive $x$ axis and the degree of orientation increased in a counterclockwise direction. In the opposite case, with the reference bar located on the right side of the body midline, the $0^{\circ}$ reference orientation was set on the negative $x$-axis and the degree of orientation increased in a clockwise direction. By applying this relative orientation coding (body-midline-related orientations), we could be certain that left and right reference bar conditions were comparable in all respects. Specifically, in this way the relation between the orientation of the reference bar and the hand orientation became identical in all conditions, regardless of the location of the reference bar with respect to the body midline. The use of absolute orientations (i.e., having the $0^{\circ}$ orientation set on the positive $x$-axis with angle increasing in a counterclockwise direction) with the whole data set would not permit this kind of aggregation. The difference between body-midlinerelated and absolute orientations will be addressed in more detail in the Results section.

The computation of the hand orientation difference was obtained by subtracting the orientation of the right hand from that of the left hand for each possible pair of reference and test bar positions. For instance, the hand orientation difference between the hands in the top box of Figure 2 would be calculated as the pointing direction of the left middle finger $\left(112^{\circ}\right)$ minus the pointing direction of the right middle finger $\left(68^{\circ}\right)$, and thus would result in a $44^{\circ}$ hand orientation difference. Smaller hand orientation differences corresponded to the pairs of reference and test bar positions at the top of the board, whereas larger hand orientation differences corresponded to the pairs at the bottom part of the board.

In the repeated measures analysis on deviations, the assumption of sphericity was tested, and where necessary, the degrees of freedom were corrected using the Greenhouse-Geisser $\varepsilon$ correction. Our regression analyses on deviations met the assumptions of linearity, of homoscedasticity, and of normally distributed residuals. Moreover, the multivariate regression analyses revealed that the assumption of no multicollinearity was also met. The minimal level of significance retained was .05 .

\section{RESULTS}

The polar plots in Figure 3 display the orientationdependent deviations, averaged over all participants and all conditions; thus, each deviation is an average over 184 measurements. The data are presented separately according to the two reference bar locations (left side vs. right side). The distance from the center of each plot specifies the amount of deviation, and the eight axes define the reference bar orientations. The gray areas display the $95 \%$ confidence interval. The point symmetry was generated by mirroring the data from the measured $0^{\circ}-157.5^{\circ}$ range in the $180^{\circ}-337.5^{\circ}$ range. A pilot experiment supports the validity of this duplication of data, since both ranges yielded the same results. The patterns of deviations make it clear why the orientation of the reference bar has to be coded in relation to the body midline and not in absolute terms: When the reference bar was located on the right side, the performance at a specific orientation (e.g., $157.5^{\circ}$ in Figure 3) could be compared with performance at the body-midline-related orientation of the reference bar on left side (i.e., $157.5^{\circ}$ in the left panel of Figure 3). As a consequence, the relation between the orientation of the 

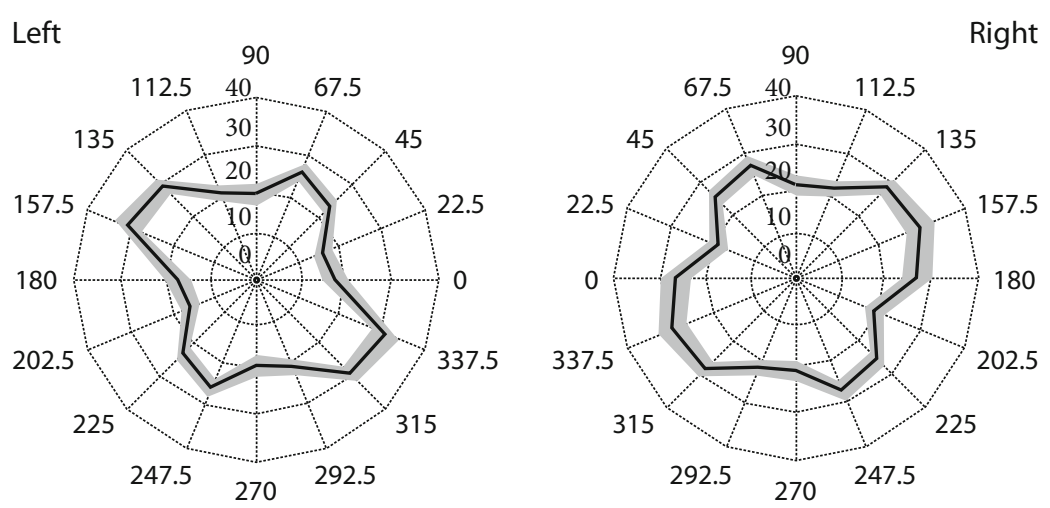

Figure 3. Polar plots of deviations, as a function of reference bar orientation for the left- and right-located reference bars, averaged over all participants and conditions. The gray areas represent the $\mathbf{9 5 \%}$ confidence interval for the standard errors of the means.

reference bar and the hand orientation was identical in the two conditions. If absolute reference orientations had been used (i.e., $0^{\circ}$ orientation on the positive $x$-axis for all data), the performances at identical orientations would correspond to different relations between the orientation of the reference bar and the hand orientation. This observation is corroborated by the following analyses, in which deviations were first analyzed with respect to the absolute reference orientation and second with respect to the body-midline-related reference orientation. The repeated measures analysis on deviations with reference bar location (left vs. right side of the board) and absolute reference bar orientation as factors revealed a significant interaction $[F(2.2,15.5)=11.373, p<.001, \varepsilon=.316]$, but no significant effect of bar location or orientation was found. In contrast, the repeated measures analysis on deviations with reference bar location (left vs. right) and bodymidline-related reference bar orientation as factors revealed a significant effect of orientation $[F(1,7)=6.963$, $p<.001]$, but neither the bar location nor, more importantly, the interaction approached statistical significance. This means that since the body-midline-related orientations of the bars gave rise to equal scanning patterns, the performance at specific reference orientations in relation to the body midline had the same magnitude, regardless of the reference bar location. Consequently, all data in the following representations and analyses were grouped over the two reference bar locations and are expressed in terms of body-midline-related reference bar orientations. Therefore, the $0^{\circ}$ orientation of the reference bar always pointed in the direction of the projection of the body midline on the frontoparallel plane, and the reference orientation angle always increased in a counterclockwise direction for the left-positioned reference bar and in a clockwise direction for the right-positioned reference bar.

In Figure 4, deviations as a function of reference bar orientation are shown for each of the 8 participants. The polar plots are sorted in ascending order of average deviation. Each deviation is an average over 46 measurements, corresponding to the 23 different combinations of bar locations and the two reference bar locations. The most important aspect of Figure 4 is that the magnitude of the deviations is clearly subject dependent. Moreover, the direction of deviations is the same for all participants; that is, the positive sign of all deviations (except for 2 out of 64 data points) reveals a systematic pattern. If the test bar is located to the right of the reference bar, the errors made are always in a clockwise direction; likewise, if the test bar is located to the left of the reference bar, the errors made are always in a counterclockwise direction. It is also worth observing how reference bar orientation influenced the performance of different participants. Whereas participants with lower average deviations manifest clear superiority in both horizontal and vertical reference bar orientations, participants with higher average deviations exhibit less pronounced orientation dependence.

On observing the confidence intervals in Figure 4, one has the impression that they are scaled with the average deviations. A one-way ANOVA revealed that standard deviations differed significantly across participants $[F(7,56)=7.893, p<.001]$. In particular, polynomial contrasts revealed a significant positive linear trend in the data $[F(1,56)=37.539, p<.001]$. Furthermore, we regressed average deviations and average standard deviations linearly, and found that the deviation was indeed a significant predictor of the standard deviation $(p<.001)$. The standard deviation could be expressed as $5.66+.14 *$ deviation $(r=.59)$; that is, standard deviations widen slightly with increasing average deviations.

To obtain a clearer view of the oblique effect (larger deviations for oblique orientations) and of the reverse oblique effect (larger deviations for cardinal orientations), and to explore how orientations exert different influences on a participant's performance, we did a more detailed analysis. In the literature, all the studies on the oblique effect have considered only the two cardinal orientations $\left(0^{\circ}\right.$ and $\left.90^{\circ}\right)$ and two oblique orientations $\left(45^{\circ}\right.$ and $\left.135^{\circ}\right)$. Accordingly, in the following analysis, the aforementioned orientations were the only ones considered. Before pooling the data, we performed two-tailed paired $t$ tests separately for each participant to assess that the performance between the two cardinal orientations and between 

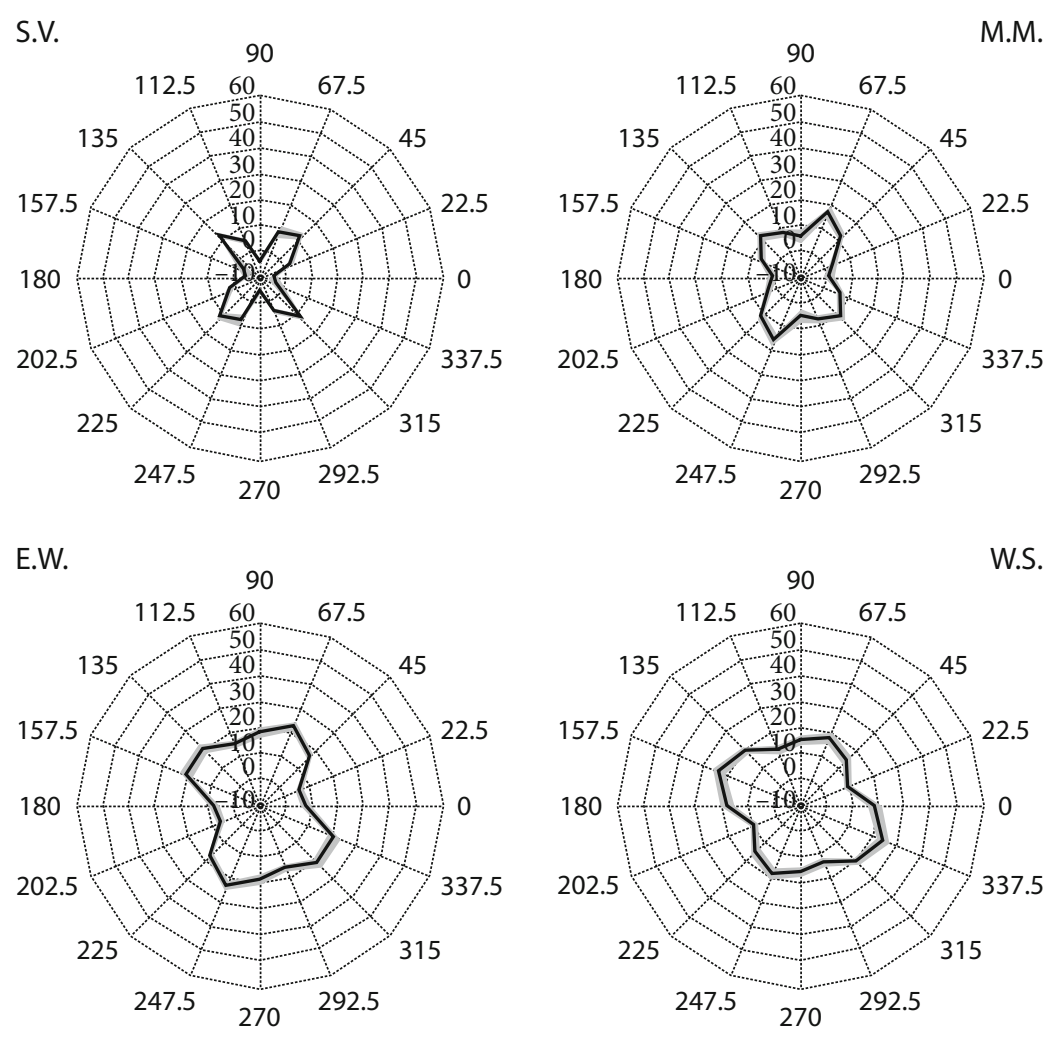

Z.H.
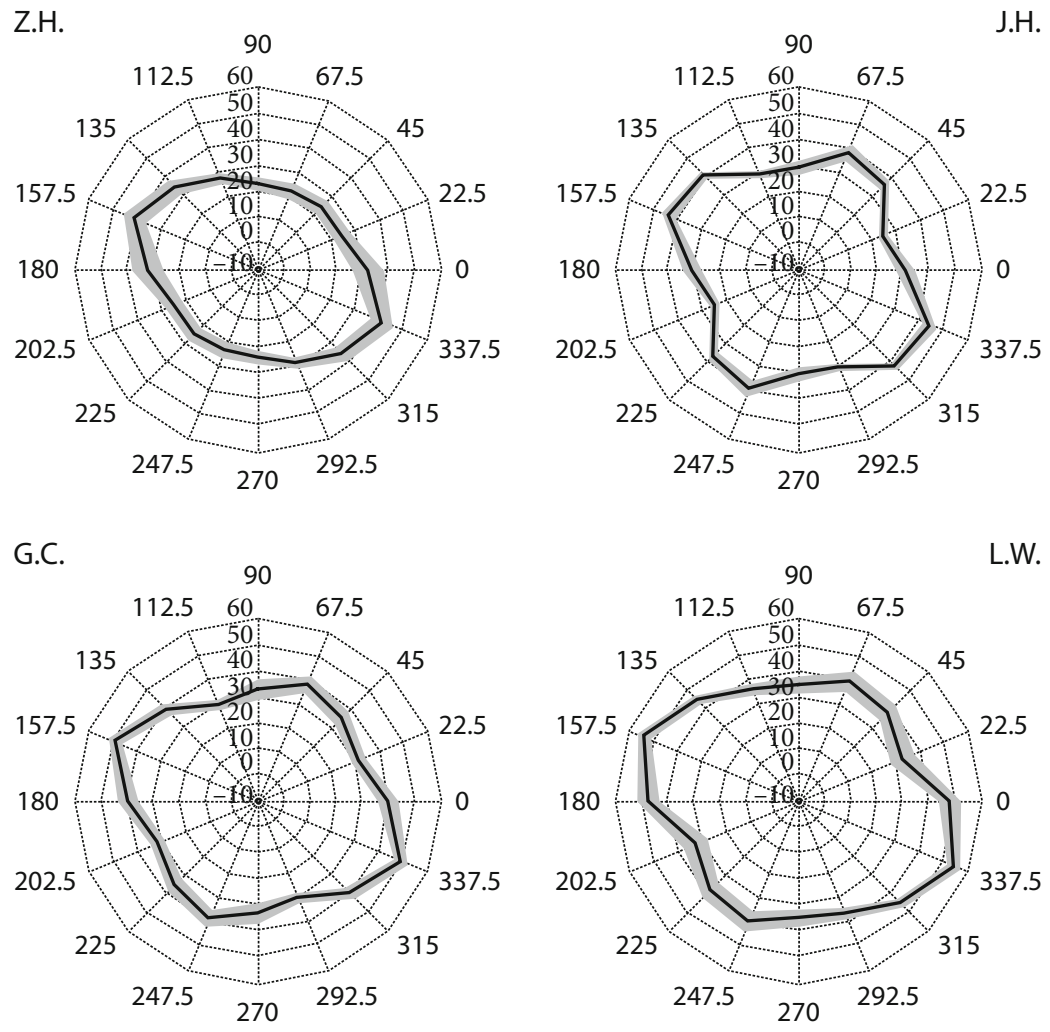

Figure 4. Polar plots of deviations as a function of reference bar orientation for the 8 participants, averaged over all conditions. The gray areas represent the $95 \%$ confidence interval for the standard errors of the means. 
the two oblique orientations did not in fact differ. Figure 5 shows a matrix of scatterplots. In each scatterplot, specific deviations - that is, deviations at the cardinal (open circles) and oblique (filled circles) orientations - are plotted for each participant against that participant's average deviation. The average deviation of each participant was calculated separately for each graph. Each data point is an average over four measurements - specifically, over those obtained for the two reference bar locations and the two reference bar orientations. Data points are fitted with the least-squares method, which illustrates highly linear increases in all scatterplots.

The whole matrix in Figure 5 represents data for 23 different combinations of reference and test bar locations. The horizontal arrangement of a scatterplot in the matrix defines the location of the reference bar, whereas the vertical arrangement determines the location of the test bar. The location of a bar is described by one parameternamely, the position of the bar specified by its height on the board (see Figure 1). This parameter comprises two

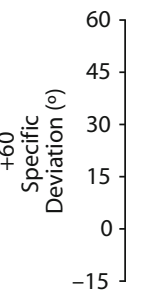
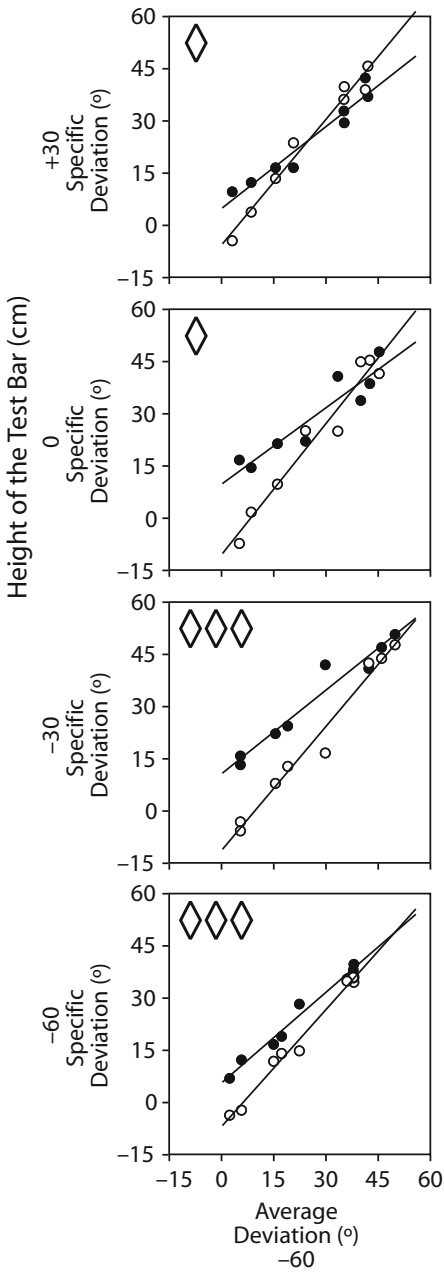

$-60$
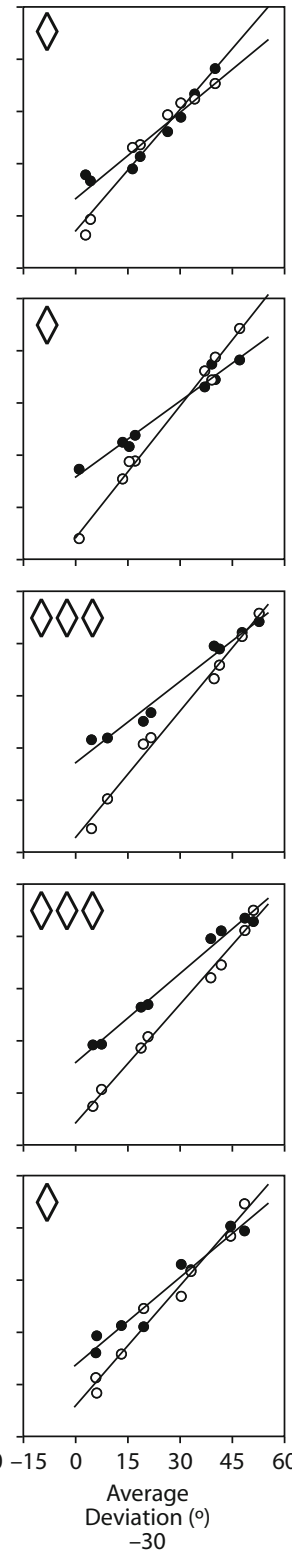
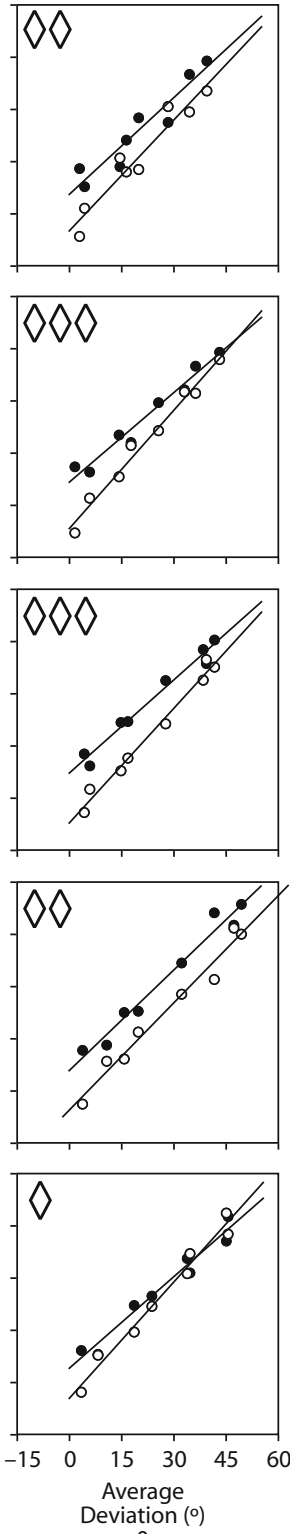

0
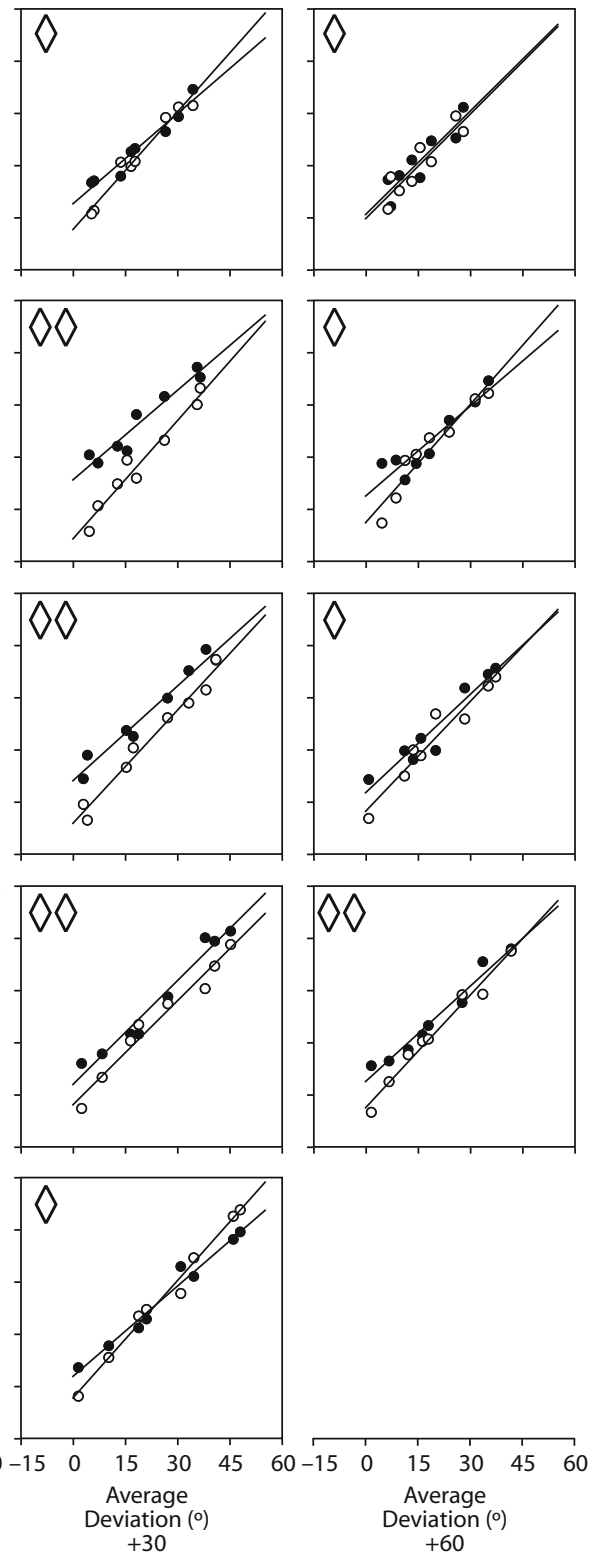

Height of the Reference Bar (cm)

Figure 5. Scatterplots of specific deviations for the cardinal $\left(0^{\circ}\right.$ and $\left.90^{\circ}\right)$ reference orientations (open circles) and the oblique $\left(45^{\circ}\right.$ and $135^{\circ}$ ) reference orientations (filled circles), plotted against average deviations. Each scatterplot displays the data of all participants for a different combination of bar locations, defined by the bar heights. Diamonds indicate the number of terms included in each best-fit regression model. 
possible bar locations, one on the left side and one on the right side of the board, as explained earlier. Since the second case is simply a mirror version of the first one, the data were pooled.

In the majority of the scatterplots, participants with moderately lower average deviations reveal a clear oblique effect, so that cardinal orientations lead to lower average deviations than oblique ones. On the other hand, participants with higher average deviations exhibit less clear distinctions, in which cardinal deviations are sometimes equal to, or even larger than, oblique deviations. Statistical analyses of the difference between cardinal and oblique deviations were performed separately on the data from each scatterplot. The factors deviation (all cardinal and oblique deviations were included, but not averaged) and obliqueness ( 1 for cardinal, -1 for oblique deviations), as well as the interaction term deviation $\times$ obliqueness, were implemented in each multiple regression model. The multiple regression models with these three terms successfully explained the data from the scatterplots marked with three diamonds in Figure 5. In these cases, the threefactors models accounted for the data significantly better than models with fewer factors, with the explained variance ranging from $96.4 \%$ to $99.1 \%$. The significance of the interaction term points to a reversal of the oblique effect; that is, participants with higher average deviations tended to perform better with oblique orientations than with cardinal orientations. Regression models without the interaction term successfully accounted for the data sets marked with two diamonds, and univariate regression models explained those data sets marked with one diamond. The explained variance ranged from $92.1 \%$ to $97.3 \%$ and from $82.2 \%$ to $96.5 \%$ for the two-predictor and one-predictor regression models, respectively. It is difficult to predict the reverse oblique effect for a specific combination of bar locations, because the prediction depends on both hand orientations as well as on both bar orientations. Moreover, the hand orientation of different participants for some particular locations could vary moderately $\left( \pm 15^{\circ}\right)$. However, by focusing attention on the combinations of bar locations that were less prone to unpredictable results, we were actually able to observe the reverse oblique effect.

In the analysis of the complete data set, it is important to consider the following two facts: namely, that the reference and test bars were located in various positions over the board and that the two bars were separated by different distances. Participants' hands thus adopted heterogeneous orientations while reaching for the bars. Therefore, if the use of an intermediate reference frame can be assumed, a hand orientation dependency could be expected, especially in the group of participants with higher average deviations. In order to test this hypothesis, hand orientation differences for the bar location combinations had to be computed by subtracting the orientation of the right hand from that of the left hand. For instance, if the middle finger of the left hand was oriented at $170^{\circ}$ and the middle finger of the right hand at $15^{\circ}$, the hand orientation difference would be $155^{\circ}$. A previous study (Kappers, 1999) showed that the magnitude of deviations is mainly influenced by the horizontal distance between bars, and in a much smaller degree by the vertical distance. No influence of position relative to the body midline was observed. To determine the distance parameters, the relative distance between the locations of the two bars was calculated. The deviation dependencies on hand orientation difference and relative distance are shown in Figure 6. Specifically, the scatterplots in the upper row represent the data of the participant with the lowest average deviation (S.V.), and those in the lower row display the data of the participant with the highest average deviation (L.W.). It can be observed unequivocally that the deviations of S.V. do not depend on the two factors; on the other hand, the deviations of L.W. increase with both hand orientation difference and distance between the bars. The data for all of the participants suggest that as average deviation increases, the tendency to depend on both factors is progressively enhanced. Stepwise regression analysis for factor selection was conducted separately on the data for each participant. We decided on a significance level of .05 in order to determine which factors to include in the models, and on a level of 1 in order to determine which to remove. The stepwise procedure showed that neither hand orientation difference nor distance had an effect on the performance of the 3 participants with lower average deviations. However, with regard to the performance of the remaining participants with higher average deviations, the stepwise regression identified hand orientation difference first ( $p<.001$ for all participants) and then distance (between $p<.001$ and $p<.01$ for different participants) as significant predictors of deviation in all cases, except for J.H., who showed only a dependence on distance $(p<.001)$.

Recently, Hermens et al. (2006) showed that deviations were larger when participants performed the parallelity task on the bottom part of the board. In the present study, it was moreover possible to examine how the reference orientation dependence combined with the effect of top versus bottom position. The data were clustered separately for each participant in two groups, according to the position of the bars on the board. When both the reference and test bars were positioned above shoulder height (the 30 - and $60-\mathrm{cm}$ heights in Figure 1), data were defined as belonging to the high group. Similarly, when both the reference and test bars were located below shoulder height (i.e., -30 and $-60 \mathrm{~cm}$ in Figure 1), the data were assigned to the low group. All other combinations of reference and test bar positions were discarded from this analysis. Twotailed paired $t$ tests on deviations were conducted separately for each participant to determine the difference in performance between the high and low groups. Deviations were significantly larger in the low group for 4 out of the 5 participants with larger average deviations. In contrast, participants characterized by smaller average deviations performed equivalently at both the top and bottom of the frontoparallel plane. The polar plots in Figure 7 represent the deviations as a function of the reference bar orientation for the high and low groups. The left polar plot shows the data of the participant with the smallest average deviation (S.V.), whereas the right plot shows the data of 

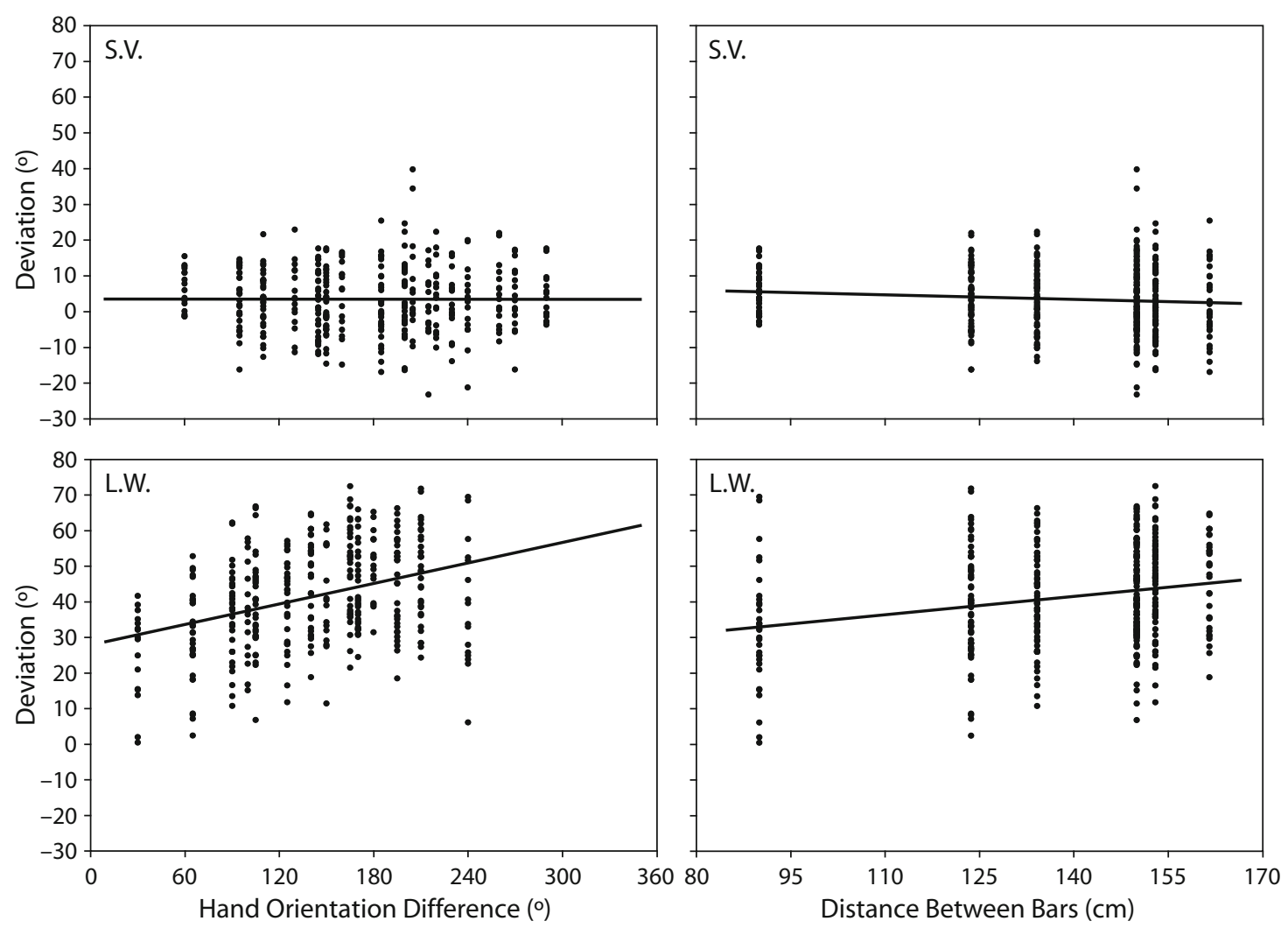

Figure 6. Scatterplots of deviations as a function of hand orientation difference (left panels) and distance between bars (right panels), for participants S.V. (top) and L.W. (bottom).

the participant with the largest average deviation (L.W.). S.V.'s deviations did not differ between the high and low groups, but on the contrary, L.W.'s deviations significantly increased (by $48.9 \%$ ) when the bars were positioned on the bottom part of the frontoparallel plane. Moreover, it is worthwhile to observe that for all participants, the relative differences in performance at different orientations were very similar, regardless of the scaling effect. Thus, the reference orientation influences performance in a consistent manner over the whole plane.

\section{DISCUSSION}

The comprehensiveness of the reference-frame-based model in explaining the origin of large deviations in the parallelity task has now also been substantiated for the frontoparallel plane. This outcome, in combination with the previously obtained results in the other two primary orthogonal planes, strongly supports the role of the egocentric and allocentric frames of reference in modulating the haptic perception of parallelity.

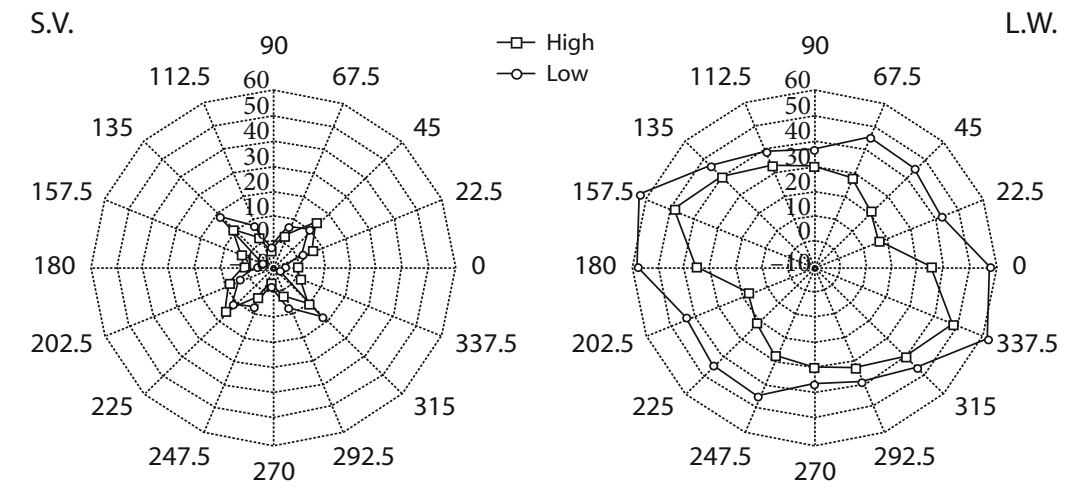

Figure 7. Polar plots of deviations as a function of reference bar orientation for participants S.V. (left) and L.W. (right). The two lines in each polar plot represent the data for combinations of bar locations corresponding to the top ("High") and bottom ("Low") parts of the frontoparallel plane. 
Just as in the parallelity tasks executed on the horizontal and midsagittal planes (Kappers, 2002, 2003, 2004, 2005; Kappers \& Koenderink, 1999), the magnitude of the deviations on the frontoparallel plane was found to be subject dependent. Despite the supposedly diverse exploratory movements that are performed on the three primary orthogonal planes, the average extent and the systematicity of deviations are fully comparable across planes. Previous studies indicated that participants who perform quite accurately probably rely more upon the allocentric reference frame. On the other hand, those who are less accurate, who can deviate by up to $90^{\circ}$ from what is geometrically parallel, can be characterized as being more egocentric. In addition, our results suggest that as the participants' average deviations increase, their standard deviations also widen accordingly. Furthermore, performance as a function of reference orientation, averaged over all stimulus locations, shows the shape of a bimodal distribution that shifts slightly as participants' average deviations increase (see Figure 4). This observation suggests that the linear increase in deviations reported by Newport et al. (2002) was due to the limited set of reference orientations used in their study.

Kappers $(2003,2004)$ and Hermens et al. (2006) reported that the oblique effect reversed for participants with larger average deviations. When physically oblique orientations are approximately aligned with or perpendicular to the hand, they turn out to be cardinal with respect to the egocentric reference frame linked to the hand. The opposite situation holds true for the physically cardinal orientations. It should be noted that an oblique effect in the previously defined egocentric reference frame would appear as a reverse oblique effect in the allocentric reference frame. In general, the preeminence of the allocentric or the egocentric reference frame for a particular participant thus determines whether the oblique effect will appear in a normal or a reverse way. In the present study, although the multifarious assortment of combinations of hand and bar orientations could confuse the predictions, our findings were consistent with previous research: The reverse oblique effect tended to emerge for participants with larger deviations. The less pronounced manifestation of the reversal in our study was mainly due to the fact that the range of deviations in the present case was smaller than in the previous studies, which mentioned that the crossover point from normal to reverse oblique effect occurred at an average deviation of about $55^{\circ}$.

A more general overview of the orientation dependence of this effect can be provided by examining how the pattern of deviations at different reference orientations was influenced by diverse positions on the frontoparallel plane. Here again, performance could be distinguished on the basis of the participants' average deviations. Only the participants characterized by larger average deviations displayed a substantial increase in deviations when both the reference and test bars were located on the bottom half of the board (see Figure 7). This scaling effect in deviations could be due to the adoption of relatively unnatural hand postures or, more probably, to the larger hand orientation differences that characterized the locations at the bottom of the board. Furthermore, it is of interest to observe that the different reference orientations had a consistent influence over the whole of the frontoparallel plane. In other words, for our given set of reference orientations, the relative differences between the deviations remained stable, regardless of the locations of the reference and test bars.

Strong support for our hypothesis of the involvement of intermediate frames of reference is supplied by the fact that the degree of hand/arm rotation correlates with the amount of deviation (Kappers, 2005). This evidence has been observed on both the horizontal and midsagittal planes in a unimanual parallelity task. It should be possible to confirm this conclusion with a bimanual task, in which the orientation difference between the two hands would be considered instead of the hand rotation. However, Hermens et al. (2006) did not detect this relationship on the frontoparallel plane. Their failure to find an association between hand orientation differences and the settings was probably caused by the limited set of bar locations used in their study. In our research, however, we used a much larger sample of bar locations. Under these conditions, we were able to determine the existence of this relationship on the frontoparallel plane. We predicted that those participants who had higher average deviations would probably be more prone to rely on the egocentric reference frame and, thus, would display stronger correlations between hand/arm orientation differences and deviations. In fact, the deviations of this class of participants in our study did indeed correlate with their hand orientation differences. An even better correspondence was obtained when the relative distance between the bar locations was included in the model. On the other hand, participants with lower average deviations, who presumably based their space representations on the allocentric frame of reference, did not exhibit any dependence, either on hand orientation differences or on relative distance between the bars.

As the main result of this study, the hypothesis that an intermediate frame of reference modulates the haptic perception of parallelity has been verified on the frontoparallel plane. Thus, the magnitude of deviations is affected by the degree to which the egocentric or the allocentric reference frame dominates. This result nicely converges with previous findings on the horizontal and midsagittal planes and reinforces the suitability of the reference-frame-based model. As a future step, it will be of extreme interest to combine the outcomes of these studies from all the primary two-dimensional orthogonal planes, in order to explore the haptic space perception of parallelity in three dimensions.

\section{AUTHOR NOTE}

This research was supported by the Netherlands Organization of Scientific Research (NWO). Correspondence relating to this article may be sent to R. Volcic, Helmholtz Institute, Utrecht University, Princetonplein 5, 3584 CC Utrecht, The Netherlands (e-mail: r.volcic@phys.uu.nl).

\section{REFERENCES}

ApPelle, S., \& Countryman, M. (1986). Eliminating the haptic oblique 
effect: Influence of scanning incongruity and prior knowledge of the standards. Perception, 15, 325-329.

Appelle, S., \& Gravetter, F. (1985). Effect of modality-specific experience on visual and haptic judgment of orientation. Perception, 14, 763-773.

Berthoz, A. (1991). Reference frames for the perception and control of movement. In J. Paillard (Ed.), Brain and space (pp. 81-111). New York: Oxford University Press.

BLUMENFELD, W. (1937). The relationship between the optical and haptic construction of space. Acta Psychologica, 2, 125-174.

Carrozzo, M., \& LaCQuaniti, F. (1994). A hybrid frame of reference for visuo-manual coordination. NeuroReport, 5, 453-456.

COREN, S. (1993). The left-hander syndrome: The causes and consequences of left-handedness. New York: Vintage.

Flanders, M., \& Soechting, J. F. (1995). Frames of reference for hand orientation. Journal of Cognitive Neuroscience, 7, 182-195.

GentaZ, E., \& Hatwell, Y. (1995). The haptic "oblique effect" in children's and adults' perception of orientation. Perception, 24, 631-646.

Gentaz, E., \& Hatwell, Y. (1996). Role of gravitational cues in the haptic perception of orientation. Perception \& Psychophysics, $\mathbf{5 8}$ 1278-1292.

Gentaz, E., \& Hatwell, Y. (1998). The haptic oblique effect in the perception of rod orientation by blind adults. Perception \& Psychophysics, 60, $157-167$.

Gentaz, E., \& Hatwell, Y. (1999). Role of memorization conditions in the haptic processing of orientations and the "oblique effect." British Journal of Psychology, 90, 373-388.

Gentaz, E., Luyat, M., Cian, C., Hatwell, Y., Barraud, P. A., \& RAPHEL, C. (2001). The reproduction of vertical and oblique orientations in the visual, haptic, and somato-vestibular systems. Quarterly Journal of Experimental Psychology, 54A, 513-526.

Gentaz, E., \& Streri, A. (2004). An "oblique effect" in infants' haptic perception of spatial orientations. Journal of Cognitive Neuroscience, 16, 253-259.

Hermens, F., Kappers, A. M. L., \& Gielen, S. C. A. M. (2006). The structure of frontoparallel haptic space is task dependent. Perception \& Psychophysics, 68, 62-75.

KAPPERS, A. M. L. (1999). Large systematic deviations in the haptic perception of parallelity. Perception, 28, 1001-1012.

KAPPERS, A. M. L. (2002). Haptic perception of parallelity in the midsagittal plane. Acta Psychologica, 109, 25-40.

KAPPERS, A. M. L. (2003). Large systematic deviations in a bimanual parallelity task: Further analysis of contributing factors. Acto Psychologica, 114, 131-145.

KAPPERS, A. M. L. (2004). The contributions of egocentric and allocentric reference frames in haptic spatial tasks. Acta Psychologica, $117,333-340$

KAPPERS, A. M. L. (2005). Intermediate frames of reference in haptically perceived parallelity. In World Haptics Conference (WHC 2005): First Joint Eurohaptics Conference and Symposium on Haptic Interfaces for Virtual Environment and Teleoperator Systems (pp. 3-11). Los Alamitos, CA: IEEE Computer Society.
Kappers, A. M. L., \& KoenderinK, J. J. (1999). Haptic perception of spatial relations. Perception, 28, 781-795.

KLATZKY, R. L. (1998). Allocentric and egocentric spatial representations: Definitions, distinctions, and interconnections. In C. Freksa, C. Habel, \& K. F. Wender (Eds.), Spatial cognition: An interdisciplinary approach to representation and processing of spatial knowledge (pp. 1-17). Berlin: Springer.

Lechelt, E. C., Eliuk, J., \& Tanne, G. (1976). Perception orientational asymmetries: A comparison of visual and haptic space. Perception \& Psychophysics, 20, 463-469.

Lechelt, E. C., \& VerenKa, A. (1980). Spatial anisotropy in intramodal and cross-modal judgments of stimulus orientations: The stability of the oblique effect. Perception, 9, 581-589.

Lederman, S. J., Klatzky, R. L., Collins, A., \& Wardell, J. (1987). Exploring environments by hand or foot: Time-based heuristics for encoding distance in movement space. Journal of Experimental Psychology: Learning, Memory, \& Cognition, 13, 606-614.

Loomis, J. M., \& Lederman, S. J. (1986). Tactual perception. In K. R. Boff, L. Kaufman, \& J. P. Thomas (Eds.), Handbook of perception and human performance: Vol. 2. Cognitive processes and performance (pp. 31.01-31.41). New York: Wiley.

Luyat, M., Gentaz, E., Corte, T. R., \& Guerraz, M. (2001). Reference frames and haptic perception of orientation: Body and head tilt effects on the oblique effect. Perception \& Psychophysics, 63, 541-554.

Millar, S., \& Al-AtTAR, Z. (2004). External and body-centered frames of reference in spatial memory: Evidence from touch. Perception \& Psychophysics, 66, 51-59.

Newport, R., RABB, R., \& JACKSON, S. R. (2002). Noninformative vision improves haptic spatial perception. Current Biology, 12, 1661-1664.

Paillard, J. (1991). Motor and representational framing of space. In J. Paillard (Ed.), Brain and space (pp. 163-182). New York: Oxford University Press.

Soechting, J. F., \& Flanders, M. (1992). Moving in three-dimensional space: Frames of reference, vectors, and coordinate systems. Annual Review of Neuroscience, 15, 167-191.

Soechting, J. F., \& Flanders, M. (1993). Parallel, interdependent channels for location and orientation in sensorimotor transformations for reaching and grasping. Journal of Neurophysiology, 70, $1137-1150$

Zuidhoek, S., Kappers, A. M. L., van der Lubbe, R. H. J., \& Postma, A. (2003). Delay improves performance on a haptic spatial matching task. Experimental Brain Research, 149, 320-330.

\section{NOTE}

1. The total number of possible combinations is 25 , but the setup used in this study did not allow including the two combinations of positions with the largest difference in height.

(Manuscript received November 4, 2005; revision accepted for publication April 26, 2006.) 